\title{
Anterior Debridement and Strut Graft with Pedicle Screw Fixation for Pyogenic Spondylitis
}

\author{
Dong-Eun Shin*, Hak-Sun Kim ${ }^{\dagger}$, Chang-Soo Ahn ${ }^{\ddagger}$, \\ Dong-Hoon Lee*, Soon-Chul Lee* \\ *Department of Orthopedic Surgery, Bundang CHA Hospital, College of Medicine, Pochon CHA University, \\ ${ }^{\dagger}$ Department of Orthopedic Surgery, Yongdong Severance Hospital, Yonsei University, College of Medicine, \\ ${ }^{\ddagger}$ Department of Orthopedic Surgery, Gumi CHA Hospital, College of Medicine, Pochon CHA University
}

Study Design: A retrospective study.

Purpose: We evaluated the results of the use of anterior debridement and interbody fusion followed by posterior spinal instrumentation.

Overview of Literature: An early diagnosis of pyogenic spondylitis is difficult to obtain. The disease can be treated with various surgical methods (such as anterior debridement and bone graft, anterior instrumentation, and posterior instrumentation).

Methods: This study included 20 patients who received anterior debridement and interbody fusion with strut bone graft followed by posterior spinal fusion for pyogenic spondylitis between 1996 and 2005. We analyzed the culture studies, the correction of the kyphotic angle, blood chemistry, the bony union period, and the amount of symptom relief.

Results: In terms of clinical symptoms relief, eight patients were grouped as "excellent", eleven patients as "good", and one patient as "fair". The vertebral body cultures were positive in 14 patients showing coagulase (-) streptococcus and S. aureus. The average times for normalization of the erythrocyte sedimentation rate and C-reactive protein level were 3.3 and 1.9 months, respectively. Four months was required for bony union. For complications, meralgia paresthetica was found in two cases.

Conclusions: Due to early ambulation and the correction of the kyphotic angle, anterior interbody fusion with strut bone graft and posterior instrumentation could be another favorable method for the treatment of pyogenic spondyulitis.

Key Words: Pyogenic spondylitis, Anterior interbody fusion, Posterior instrumentation

\section{Introduction}

Surgical treatments such as anterior debridement and bone graft, anterior instrumentation, and either single or combined posterior instrumentation ${ }^{1-3}$ have been introduced as well as other techniques (i.e. percutaneous drainage and irrigation $^{4}$, percutaneous transpedicular discectomy ${ }^{5}$ and percutaneous spinal external fixation ${ }^{6}$. The most effective of the various surgical methods to eradicate infection has not been evaluated. We analyzed patients that underwent anteri- or debridement and fusion followed by posterior instrumentation.

\section{Materials and Methods}

\section{Patients}

This study included 20 patients with pyogenic spondylitis who underwent anterior debridement and interbody fusion with strut bone graft followed by posterior instrumentation from 1996 to 2005. The mean age of the patients was 59

Corresponding author: Dong-Eun Shin, MD

Department of Orthopedic Surgery, Bundang CHA Hospital, College of Medicine, Pochon CHA University

351 Yatap-dong, Bundang-gu, Seongnam, 463-712, Korea

Tel: +82-31-780-5289, Fax: +82-31-708-3578, E-mail: shinde@cha.ac.kr 
years (range, 38 80 years) and the mean follow-up period was 25 months (range, 12 42 months). Accompanying diseases were hypertension in four patients, diabetes mellitus in three patients, and other disorders including hyperthyroidism, acute glomerulonephritis, and dementia. Three Lesions were in the thoracic region, 4 legions in the thoracolumbar region and 13 lesions in the lumbosacral region. Neurological deficits were present in 10 cases prior to surgery. The mean time to diagnosis was 5 weeks. The extent of surgery was determined based on diagnosis made by MRI. The indications for surgical debridement included neurological compromise, failed medical treatment, soft tissue extension, extensive vertebral body and disc space destruction, and a progressive deformity'.

\section{Methods}

A retrospective analysis of the patient medical records and imaging studies were performed along with a telephone survey. The clinical results and the neurological changes were assessed according to the McNab classification (Table 1) and ASIA impairment scale, respectively. The degree of bony union was investigated using the Lenke classification (Table 2). Cobb's method was used for measuring changes in the kyphotic angle when imaging studies showed complete bony union. The duration for normalization of the hematological parameters, i.e. the white blood cell (WBC) count, the erythrocyte sedimentation rate (ESR) and C-reac- tive protein (CRP) level, were recorded. Culture studies for microorganisms were performed after surgery. The metal failure or screw loosening, and other neurological injuries were also analyzed.

\section{Surgical technique and postoperative care}

To decide the level of posterior instrumentation preoperatively, if a large part of the vertebral bodies were involved with signal changes as seen on MRI, then instrumentation was performed that extended to one level above and below the involved vertebrae. If only a part of a vertebrae showed signal changes, and pedicle fixation was feasible, then instrumentation was performed on the most proximal and distal affected segments. For the level of anterior fusion, a strut bone graft was performed followed by a corpectomy on all vertebral bodies that showed signal changes on MRI preoperatively. If the lesion was in only one vertebra, a strut bone graft was performed toward just above or below the end plate of the more destructed part.

We performed the procedure under general anesthesia with the patient in the prone position. We dissected subperiostealy using Cobb's elevator down the spinous process and along the laminar to the facet joint. Using an image intensifier, pedicle screws were inserted to both sides of the pedicles at the lesion by use of the intersection technique. Then a rod was inserted to the pedicle screws.

Next, the patient was placed in the semi-lateral position

Table 1. MacNab classification

\begin{tabular}{ll}
\hline Grade & Description \\
\hline $\begin{array}{l}\text { Excellent } \\
\text { Good }\end{array}$ & No pain; no restriction of activity \\
& $\begin{array}{c}\text { Occasional back or leg pain of sufficient severity to interfere with the patient's ability to do his nor- } \\
\text { mal work or his capacity to enjoy himself in his leisure hours. } \\
\text { Fair }\end{array}$ \\
Improved functional capacity, but handicapped by intermittent pain of sufficient severity to curtail or \\
moor \\
mo improvement or sufficient improvement to enable increase in activities; further operative interven- \\
tion required.
\end{tabular}

Table 2. Assessment of anterior fusion grades (Lenke)

\begin{tabular}{ll}
\hline Grade & Description \\
\hline 1 & Fused with remodeling and trabeculae \\
2 & Graft intact, not fully incorporated; no lucencies \\
3 & Graft intact with definite proximal or distal lucencies \\
4 & Graft broken or resorbed \\
\hline
\end{tabular}


with the right side exposed. The infected lesion was approached with a transthoracic or retroperitoneal approach. Curettage and debridement was performed with a corpectomy. A strut bone graft was performed from the rib or iliac crest. The iliac crest donor site was reconstructed by use of a $1 / 3$ tubular plate? There was one case where a mesh was inserted. The drain was removed when the amount was below $50 \mathrm{cc}$. Ambulation was started at the next day with the use of a brace (Figs. 1-3). For two cases out of the total 20 cases, hardware was removed at 2 years postoperatively.

\section{Results}

\section{Clinical result (Table 3)}

For clinical symptom relief, seven patients were grouped as 'excellent,' twelve patients 'good,' and one patient as 'fair.' Half of the 10 patients that had a neurological deficit prior to surgery were improved in motor and sensory function after surgery. With regards to the time to diagnosis, logistic regression analysis showed a significant difference $(\mathrm{p}=0.05)$ between patients with or without positive improvement.
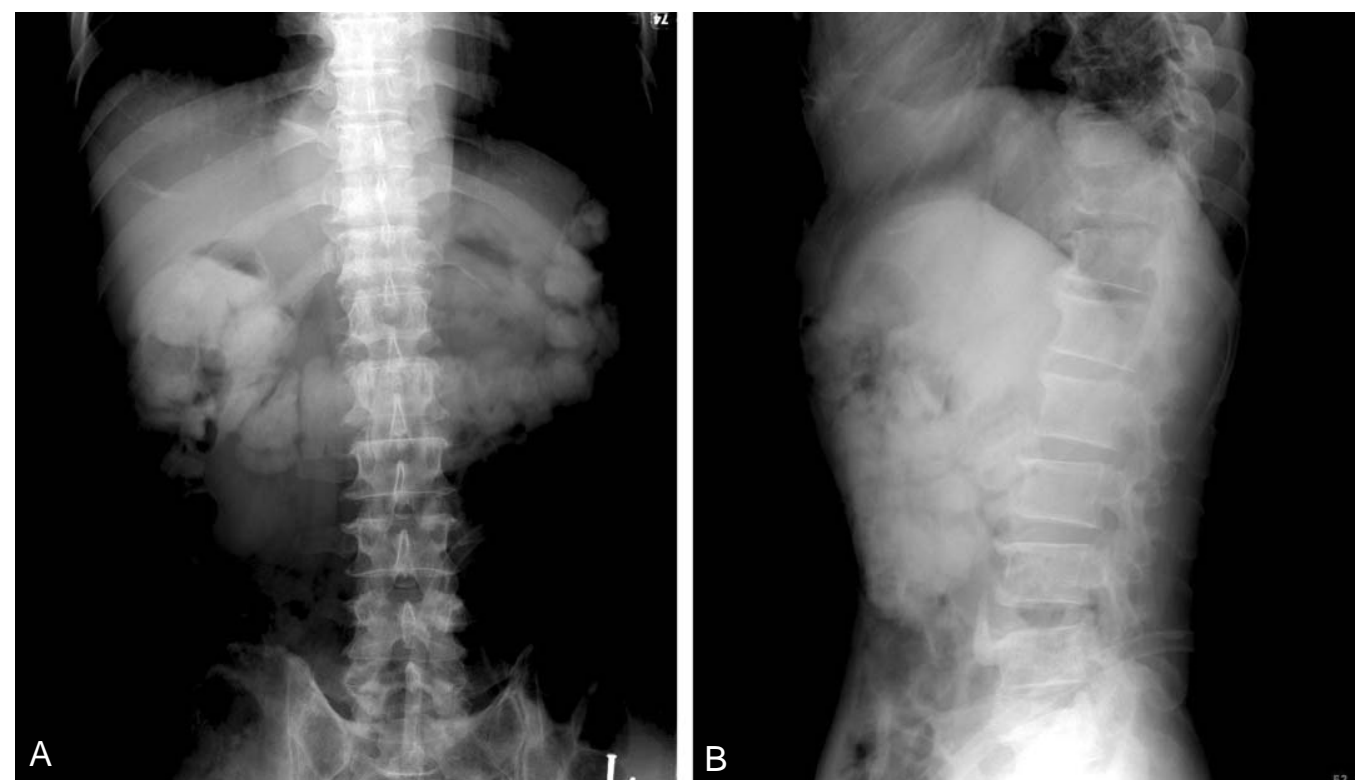

Fig. 1. Anterior wedge compressive deformity is shown on T11, T12 vertebral body. (A) Anterior posterior view. (B) Lateral view.
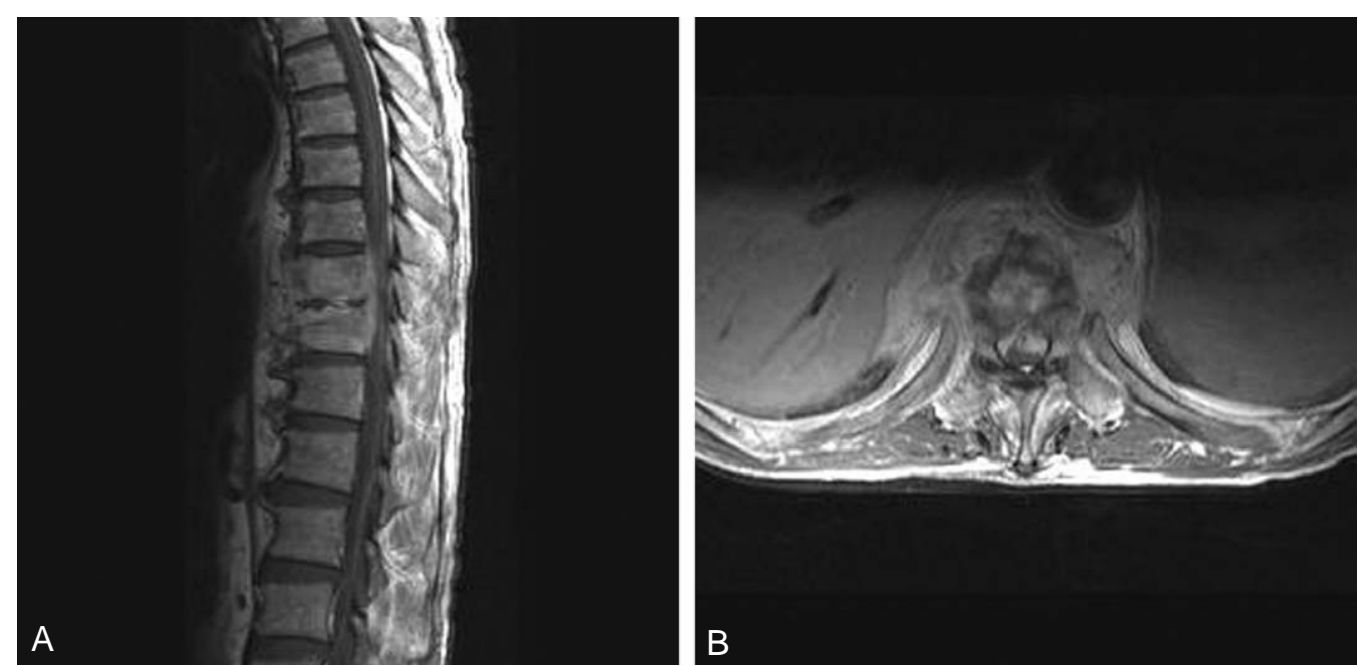

Fig. 2. T-L-spine MRI, 64-year-old male patient. (A) In the axial view, infectious spondylitis and discitis with paravertebral abcess is shown. (B) In the sagittal view, the prevertebral abcess and infectious spondylitis T11 and T12. 


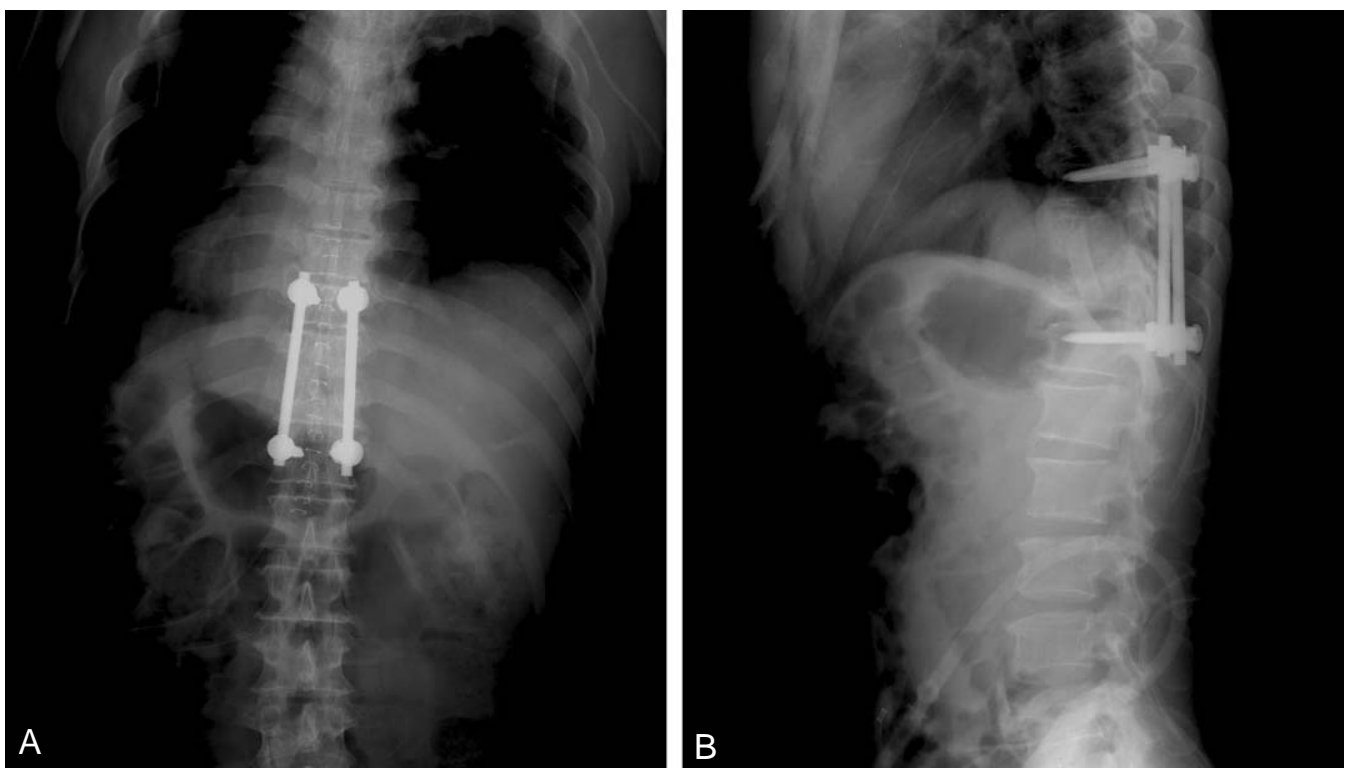

Fig. 3. The kyphotic angle is recovered with the posterior pedicle screw instrumentation and anterior interbody fusion with auto iliac bone graft from L1 to L4. (A) Anterior posterior view. (B) Lateral view.

Table 3. Demographic data and result

\begin{tabular}{|c|c|c|c|c|c|c|c|c|}
\hline No & Sex & Age & Fusion level post & Fusion level ant & Lesion & $\mathrm{McNab}$ & Lenke & Neurologic deficit change \\
\hline 1 & $\mathrm{M}$ & 80 & $\mathrm{~L} 3,4,5$ & $\mathrm{~L} 3,4$ & L4 & $\mathrm{F}$ & 2 & Unchanged \\
\hline 2 & $\mathrm{M}$ & 38 & $\mathrm{~L} 1,2,3,4$ & $\mathrm{~L} 2,3$ & $\mathrm{~L} 2,3$ & $\mathrm{E}$ & 1 & Preop none \\
\hline 3 & $\mathrm{M}$ & 62 & $\mathrm{~L} 1,2,3,4$ & L3, 4 & L3, 4 & E & 1 & Improved \\
\hline 4 & M & 60 & $\mathrm{~T} 9,10,11$ & $\mathrm{~T} 10,11$ & $\mathrm{~T} 10,11$ & G & 1 & Preop none \\
\hline 5 & $\mathrm{~F}$ & 67 & $\mathrm{~L} 1,2,3$ & $\mathrm{~L} 2,3$ & $\mathrm{~L} 2,3$ & G & 1 & Improved \\
\hline 6 & M & 67 & $\mathrm{~L} 1,2,3$ & $\mathrm{~L} 2,3$ & $\mathrm{~L} 2,3$ & G & 1 & Preop none \\
\hline 7 & $\mathrm{M}$ & 42 & $\mathrm{~L} 4,5, \mathrm{~S} 1$ & L5, S1 & L5, S1 & $\mathrm{E}$ & 1 & Preop none \\
\hline 8 & $\mathrm{~F}$ & 75 & $\mathrm{~L} 3,4,5$ & $\mathrm{~L} 3,4$ & $\mathrm{~L} 3,4$ & G & 1 & Preop none \\
\hline 9 & $\mathrm{~F}$ & 80 & $\mathrm{~L} 3,4$ & $\mathrm{~L} 3,4$ & L4 & G & 2 & Improved \\
\hline 10 & $\mathrm{M}$ & 56 & $\mathrm{~L} 1,2,3,4$ & $\mathrm{~L} 3,4$ & $\mathrm{~L} 3,4$ & G & 1 & Preop none \\
\hline 11 & $\mathrm{M}$ & 69 & $\mathrm{~L} 1,2,3$ & $\mathrm{~L} 1,2$ & $\mathrm{~L} 1,2$ & $\mathrm{E}$ & 2 & Unchanged \\
\hline 12 & $\mathrm{~F}$ & 81 & $\mathrm{~T} 9,10,11$ & $\mathrm{~T} 10,11$ & $\mathrm{~T} 10,11$ & G & 1 & Preop none \\
\hline 13 & $\mathrm{~F}$ & 56 & $\mathrm{~T} 8,9$ & $\mathrm{~T} 8,9$ & $\mathrm{~T} 8,9$ & E & 1 & Unchanged \\
\hline 14 & $\mathrm{M}$ & 44 & L4, 5, S1 & L5, S1 & L5, S1 & E & 1 & Unchanged \\
\hline 15 & $\mathrm{~F}$ & 57 & L3, 4 & L3, 4 & $\mathrm{~L} 3,4$ & G & 1 & Improved \\
\hline 16 & $\mathrm{~F}$ & 82 & L3, 4 & L3, 4 & L4 & G & 2 & Preop none \\
\hline 17 & M & 65 & $\mathrm{~L} 2,3,4$ & L3, 4 & L3, 4 & G & 1 & Preop none \\
\hline 18 & M & 70 & L5, S1 & L5, S1 & L5, S1 & E & 2 & Unchanged \\
\hline 19 & $\mathrm{~F}$ & 80 & $\mathrm{~T} 9,10,11$ & $\mathrm{~T} 10,11$ & $\mathrm{~T} 10,11$ & G & 1 & Preop none \\
\hline 20 & M & 56 & $\mathrm{~L} 3,4$ & $\mathrm{~L} 2,3$ & L3 & G & 1 & Improved \\
\hline
\end{tabular}

\section{Imaging studies (Table 4)}

To obtain complete bony unions as seen on the plain radiographs, an average of 4 months was required. Fifteen cases of grade 1 and 5 cases of grade 2 were identified by Lenke's classification. The kyphotic angle was measured for all but one case involving the upper thoracic vertebrae. The average correction angle was 5 degrees after surgery.

\section{Laboratory results}

Before surgery, WBC counts in 9 patients were $10000 / \mu \mathrm{L}$ 
Table 4. Kyphosis angle

\begin{tabular}{rrrrr}
\hline No & Preop kyphosis angle & Immediate postop kyphosis angle & Last f/up kyphosis angle & Kyphosis angle correction \\
\hline 1 & 13 & 8 & -7 & 20 \\
2 & 15 & 7 & -10 & 25 \\
3 & -5 & -10 & 7 & -12 \\
4 & -12 & -17 & -16 & 4 \\
5 & 8 & 3 & 1 & 7 \\
6 & -10 & -3 & 4 & -14 \\
7 & 7 & 5 & 11 & -4 \\
8 & 13 & 7 & 3 & 10 \\
9 & 12 & 4 & -1 & 13 \\
10 & -5 & -7 & 10 & -15 \\
11 & -10 & -13 & 2 & -12 \\
12 & 1 & -6 & -15 & 16 \\
13 & -15 & -15 & -16 & 1 \\
14 & 8 & 2 & 14 & -6 \\
15 & -3 & 0 & 5 & -8 \\
16 & 14 & -2 & -13 & 27 \\
17 & -2 & -5 & -7 & 5 \\
18 & 11 & 6 & -3 & 14 \\
19 & 12 & 6 & -3 & 15 \\
20 & 15 & 8 & 1 & \\
\hline
\end{tabular}

or more and the average was $9700 / \mu \mathrm{L}$. All patients showed normalization of the WBC counts within 4 months after surgery. For the ESR, with a normal range of $0 \sim 10 \mathrm{~mm} / \mathrm{hr}$ in men and 0 20 mm/hr in women, became normalized in 9 patients after 3 months on average. The other 11 patients showed a decrease in the ESR from $65.8 \mathrm{~mm} / \mathrm{hr}$ preoperatively to $44.2 \mathrm{~mm} / \mathrm{hr}$ postoperatively on average. The preoperatively checked CRP level was $6.0 \mathrm{mg} / \mathrm{dL}$ on average. The average duration for the CRP level to become normalized was 1.9 months. In two patients, the CRP level remained above $10 \mathrm{mg} / \mathrm{dL}$ (11.4 and $8.5 \mathrm{mg} / \mathrm{dL}$, respectively). In 14 cases, culture studies showed the presence of $S$. aureus in half of the cases and coagulase (-) staphylococci in the other half of the cases.

\section{Other complications}

There was one case of incomplete bony union, which was later corrected by a subsequent revision operation. A complete bony union was finally achieved, as seen on follow-up $\mathrm{X}$-rays. Two cases of sensory disturbance in the femoral area were reported but the patients complained of minimum interference in daily activities.

\section{Discussion}

In 1911, Albee $^{8}$ proposed posterior instrumentation with auto transplantation of the tibia for treatment of tuberculous spondylitis, which marked progression of surgical treatment ${ }^{9}$. Since Hodgson and Stock ${ }^{10}$ proposed anterior fusion (instrumentation) with anterior debridement and auto transplantation in 1960, its use has gained wide popularity although posterior instrumentation was added to the procedure for fast rehabilitation. Wang ${ }^{11}$ has stated that physiologically the anterior and middle spinal column could bear $70 \sim 80 \%$ of the axial gravitational pressure and the posterior column could bear the remaining 20 30\%. On that basis, Wang advocated the use of the anterior fusion method with an anterior approach, arguing that present day posterior instrumentation alone without anterior instrumentation could not bear a pressure of $1,300 \mathrm{~N}$, physiologically exerted to the anterior column.

Also suggested was a method of posterior approach where laminectomy and resection of granulation tissue protruded into epidural space are performed and then are supplemented by posterior fusion with preceding bone transplantation on the co-resected disc area ${ }^{12}$. However, this method is useful with only one body infection; in our study 
this situation was found in only two cases among the 20 patients. We think this method is a somewhat limited technique.

One of the new minimally invasive surgical treatments is percutaneous transpedicular discectomy. This method allows both definitive diagnosis and treatment at the same time and could be used for cases in the early stage where there is no instability, bone destruction or other neurological symptoms ${ }^{5}$.

Another method is percutaneous drainage and irrigation ${ }^{4}$, which was suggested by Hanaoka and colleagues. This is reserved as a second line treatment for patients with a poor general condition, evidence of an abscess on MRI, and advanced bone destruction.

Some surgeons perform posterior instrumentation 1 2 weeks after anterior fusion. In contrast, we performed anterior debridement and fusion immediately followed by posterior spinal instrumentation ${ }^{13}$.

Sensory disturbance in one patient, who was reported as 'fair' according to McNab's classification for the clinical result, was found in the femoral area where the bone graft was harvested, implying no correlation with symptoms of pyogenic spondyiltis. One other patient who reported such a sensory disturbance complained only of minimal interruption in daily life during the one-year postoperative period. A preoperative neurological deficit could not be assessed in two patients due to dementia in both cases but could be found in 10 other patients. Of these 10 cases, half of the patients showed improvement postoperatively. As reported in the result, there was significant correlation the time until diagnosis with neurological improvement. For this reason, we believe that early decompression is important. For blood chemistry, the ESR and CRP level decreased significantly within 3.3 and 1.9 months respectively. Two patients showed persistently high CRP levels postoperatively but the high levels could be attributed to the fact that one patient developed a bed sore after admission to the hospital and the other patient developed cellulitis in the right foot. A culture for microorganisms is known to yield positive results in $60 \sim 80 \%$ of cases in general ${ }^{14}$, which was confirmed in this study, yielding 14 positive results ( 7 cases of $S$. aureus infection and 7 cases of coagulase (-) staphylococci infection) out of 20 cases. S. govender said that the frequent organism is $S$. aureus, Streptococcus, and coagulase (-) staphylococci are assumed to cause subclinical infections that probably account for a low ESR and a decreased CRP level ${ }^{15}$.

\section{Conclusions}

As early ambulation and the correction of the kyphotic angle are possible, anterior interbody fusion with a strut bone graft and posterior instrumentation could be another favorable method for the treatment of pyogenic spondyulitis.

\section{REFERENCES}

1. Kim EH, Lee JH, Shin DE, Wang WJ, Kim HM, Tak HC: Anterior debridement and strut graft with pedicle screw fixation for tuberculous spondylitis. J Korean Soc Spine Surg 2005; 12: 358-364.

2. Ahn DK, Jeong KW, Kwon KB, Cha SK, Park KY, Cho KH: Operative treatment of lumbosacral spondylitis through a posterior-only approach. J Korean Orthop Assoc 2005; 40: 868-874.

3. Choy WS, Kim WJ, Lee KW, et al: A comparative study of anterior interbody fusion with and without posterior instrumentation in infection of the lumbar spine. J Korean Soc Spine Surg 1999; 6: 458-463.

4. Hanaoka N, Kawasaki Y, Sakai T, et al: Percutaneous drainage and continuous irrigation in patients with severe pyogenic spondylitis, abcess formation, and marked bone destruction. J Neurosurg Spine 2006; 4: 374-379.

\section{Hadjipavlou AG, Katonis PK, Gaitanis IN, Muffoletto} AJ, Tzermiadianos MN, Crow W: Percutaneous transpedicular discectomy and drainage in pyogenic spondylodiscitis. Eur Spine J 2004; 13: 707-713.

6. Jeanneret B, Magerl F: Treatment of osteomyelitis of the spine using percutaneous suction/irrigation and percutaneous external spinal fixation. J Spinal Disord 1994; 7: 185-205.

7. Shin DE, Cho DY, Han SH, Kim JH, Lee SC: Reconstruction of Iliac Crest Donor Site Using 1/3 Tubular Plate. J Korean Soc Spine Surg 2006; 13: 87-92.

8. Albee FH: Transplantation of a portion of the tibia into the spine for Pott's disease: a preliminary report 1911. Clin Orthop Relat Res 2007; 460: 14-16

9. Calderone RR, Larsen JM: Overview and classification of spinal infections. Orthop Clin North Am 1996; 27: 1-8.

10. Hodgson AR, Stock FE: Anterior spinal fusion a preliminary communication on the radical treatment of Pot's disease and Pott's paraplegia. Br J Surg 1956; 44: 266-275.

11. Wang JM: Anterior interbody fusion and posterior instru- 
mentation. J Korean Soc Spine Surg 2005; 12: 159-163.

12. Swanson AN, Pappou IP, Cammisa FP, Girardi FP: Chronic infections of the spine: surgical indications and treatments. Clin Orthop Relat Res 2006; 444: 100-106.

13. Dimar JR, Carreon LY, Glassman SD, Campbell MJ, Hartman MJ, Johnson JR: Treatment of pyogenic vertebral osteomyelitis with anterior debridement and fusion followed by delayed posterior spinal fusion. Spine 2004; 29:
326-332.

14. Chang MC, Wu HT, Lee CH, Liu CL, Chen TH: Tuberculous Spondylitis and pyogenic spondylitis: comparative magnetic resonance imaging features. Spine 2006; 31: 782788.

15. Govender S: Spinal infections. J Bone Joint Surg Br 2005; 87B: 1454-1458. 